\title{
Influence of stratification duration and hormone concentration in aonla (Emblica officinalis, GAERTN.)
}

Surya Narayan

Department of Horticulture, K.A.P.G., Collage Allahabad, 211001, (India)

Email: sunara.kapg@gmail.com

\begin{abstract}
The experiment was conducted at the Department of Horticulture, Kulbhasker Ashram Post Graduate Collage, Allahabad, Uttar Pradesh with a view to standardize suitable stratification duration and hormone concentration for Aonla seed treatment. There were ten treatment combinations $\left(\mathrm{T}_{1}\right.$ to $\left.\mathrm{T}_{10}\right)$ including a control. Different duration of seed stratification i.e., 24hours, 48 hours and 72 hours were tried along with the 100ppm,200ppm and $300 \mathrm{ppm} \mathrm{GA}_{3}$ seed treatment. Treated seeds were sown in the polythene bags $(25 \times 15 \mathrm{~cm}$ size, 200 gauge thick) containing soil, sand and FYM mixture (1:1:1). It was interesting to note that the effect of stratification duration and hormone treatment concentration was found to be significant for seed germination, transplanting success, seedling mortality percentage and rate of seed germination. Treatment $\mathrm{T}_{6}(48 \mathrm{hrs}+300 \mathrm{ppmGA} 3)$ yielded highest percentage, $(84.00)$ of seed germination while the lowest percentage value (37.25) was recorded in $\mathrm{T}_{9}$ (72hrs+300ppmGA3) treatment and the transplanting success was also lowest in $\mathrm{T}_{9}$. The seedling mortality percentage was maximum (79.25) with $\mathrm{T}_{9}$ where as lowest percentage value (22.00) was observed for $\mathrm{T}_{6}$ treatment. It may be concluded that $\mathrm{T}_{6}$ treatment can be recommended for the better stand establishment of Aonla nursery.
\end{abstract}

Key Words: Stratification, Treatment, $\mathrm{GA}_{3}$, Nursery, Aonla, Mortality, Seedling, Germination, Seed.

Paper Cited: Narayan, S. (2017). Influence of stratification duration and hormone concentration in aonla (Emblica officinalis, GAERTN.). South Asian Journal of Food Technology and Environment, 3(2): 581-584.

\section{Introduction}

Increased demand of aonla (Emblica officinalis, Garten.) buddlings in traditional as well as nontraditional areas of India due to its peculiar character of diverse use, medicinal value, tolerance to biotic and abiotic stresses, higher benefit cost ratio and positive government policies emphasized to chalk out some feasible and acceptable measures for the better stand-establishment of saplings at the nursery stage. Aonla buddlings are prepared thorough budding on seedling root - stock which is obtained through seeds. In nature, aonla seed has poor germination and higher seedling mortality, owing to adverse edaphic conditions during nursery stage. Therefore it becomes imperative to standardize suitable stratification time and exact hormone concentration for seed treatment for flourishing the aonla nursery-industry. Certainly, these tactics are the most important component to provide sound base for propagation, once time and concentration is standardize, we shall be able to grow healthy seedlings with faster rate.

Keeping these aspects in view, the experiment was under taken to ascertain the effect of the stratification and hormone treatment on seed germination, rate of seed germination, transplanting success and mortality of seedlings.

\section{Materials and Method}

The experiment was conducted at the Department of Horticulture, Kulbhasker Ashram Post Graduate Collage, Allahabad, Uttar Pradesh during the year 2012-13 with a view to standardize suitable stratification duration and hormone concentration for Aonla seed treatment. There were ten treatment combinations $\left(T_{1}\right.$ to $\left.T_{10}\right)$ including a control. Different duration of seed stratification i.e., 24hours, 48 hours and 72 hours were tried. Soaked seed were put in layers under different strata of moist sand for varying duration. $\mathrm{GA}_{3}$ hormone @ 100ppm, $200 \mathrm{ppm}$ and $300 \mathrm{ppm}$ was used for seed treatment after stratification. Treated seeds were sown in the polythene bags $(25 \times 15 \mathrm{~cm}$ 
size, 200 gauges thick) containing soil, sand and FYM mixture $(1: 1: 1)$.

\section{Results and Discussion}

Seed germination in aonla started after 3 days of seed sowing and completed within 27 days in all the treatment. Seed germination under different treatments ranged between 37.25 to 84.00 percent. The percentage of seed germination as influenced by treatments differed significantly. The maximum seed germination $(84.00 \%)$ was recorded in treatment $\mathrm{T}_{6}(48 \mathrm{hrs}$ stratification $+300 \mathrm{ppm}$ $\mathrm{GA}_{3}$ ) which was significantly superior to all other treatments and the value was lowest $(37.25 \%)$ in $\mathrm{T}_{9}(72 \mathrm{hrs}$ stratification $+300 \mathrm{ppm}$
$\left.\mathrm{GA}_{3}\right)$. The findings of the study supported and corroborated the findings of Bisla et al., (1984) in Ber and Govind and Chandra (1993) in Khasi Mandrin. The lowest percentage of seed germination obtained with treatment $\mathrm{T} 9$ indicated adverse effect of longer duration of stratification coupled with toxic concentration $\mathrm{GA}_{3}$ which augmented seed decay and partial damage of seed too. Over tendering of seed coat and ultra concentration of $\mathrm{GA}_{3}$ might be corroded the plume and radicle of the seed resulting failure of germination. The possibility of exo-osmosismay not be denied. Dewey (1960); Paliwal and Gandhi (1968) and Ayers and Westcot (1976) also observed the same causes.

Table 1: Influence of stratification duration and hormone concentration on seed germination and rate of seed germination in Aonla (Emhlica officinalis, GAERTN.)

\begin{tabular}{|c|c|c|c|c|c|c|c|c|c|}
\hline \multirow{2}{*}{$\begin{array}{l}\text { Treat- } \\
\text { ments }\end{array}$} & \multicolumn{8}{|c|}{ Seed germination $(\%)$} & \multirow{2}{*}{$\begin{array}{l}\text { Rate of seed } \\
\text { germination }\end{array}$} \\
\hline & $\begin{array}{c}3 \\
\text { DAS }\end{array}$ & $\begin{array}{c}6 \\
\text { DAS }\end{array}$ & $\begin{array}{c}9 \\
\text { DAS }\end{array}$ & $\begin{array}{c}12 \\
\text { DAS }\end{array}$ & $\begin{array}{c}15 \\
\text { DAS }\end{array}$ & $\begin{array}{c}18 \\
\text { DAS }\end{array}$ & $\begin{array}{c}21 \\
\text { DAS }\end{array}$ & $\begin{array}{c}27 \\
\text { DAS }\end{array}$ & \\
\hline $\mathrm{T}_{1}$ & $\begin{array}{c}2.95 \\
(9.89)\end{array}$ & $\begin{array}{c}22.66 \\
(23.29)\end{array}$ & $\begin{array}{l}43.33 \\
(35.4)\end{array}$ & $\begin{array}{c}48.33 \\
(37.06)\end{array}$ & $\begin{array}{c}53.66 \\
(40.92)\end{array}$ & $\begin{array}{c}56.00 \\
(45.12)\end{array}$ & $\begin{array}{c}56.00 \\
(45.12)\end{array}$ & $\begin{array}{c}56.00 \\
(45.12)\end{array}$ & 10.43 \\
\hline $\mathrm{T}_{2}$ & $\begin{array}{c}3.05 \\
(10.3)\end{array}$ & $\begin{array}{c}22.66 \\
(25.29)\end{array}$ & $\begin{array}{c}45.33 \\
(38.40)\end{array}$ & $\begin{array}{c}50.33 \\
(40.06)\end{array}$ & $\begin{array}{c}58.66 \\
(45.92)\end{array}$ & $\begin{array}{c}60.00 \\
(50.12)\end{array}$ & $\begin{array}{c}60.00 \\
(50.12)\end{array}$ & $\begin{array}{c}60.00 \\
(50.12)\end{array}$ & 10.25 \\
\hline $\mathrm{T}_{3}$ & $\begin{array}{c}3.25 \\
(10.30)\end{array}$ & $\begin{array}{c}24.66 \\
(28.29)\end{array}$ & $\begin{array}{l}47.33 \\
(40.4)\end{array}$ & $\begin{array}{c}53.33 \\
(45.06)\end{array}$ & $\begin{array}{c}60.66 \\
(48.92)\end{array}$ & $\begin{array}{c}61.00 \\
(52.12)\end{array}$ & $\begin{array}{c}61.00 \\
(52.12)\end{array}$ & $\begin{array}{c}61.00 \\
(52.12)\end{array}$ & 10.01 \\
\hline $\mathrm{T}_{4}$ & $\begin{array}{c}2.36 \\
(8.83)\end{array}$ & $\begin{array}{c}25.66 \\
(30.29) \\
\end{array}$ & $\begin{array}{l}49.33 \\
(44.4)\end{array}$ & $\begin{array}{c}55.33 \\
(48.06) \\
\end{array}$ & $\begin{array}{c}63.66 \\
(52.92) \\
\end{array}$ & $\begin{array}{c}64.00 \\
(53.12) \\
\end{array}$ & $\begin{array}{c}64.00 \\
(53.12)\end{array}$ & $\begin{array}{c}64.00 \\
(53.12)\end{array}$ & 11.40 \\
\hline $\mathrm{T}_{5}$ & $\begin{array}{c}3.60 \\
(10.82)\end{array}$ & $\begin{array}{c}25.66 \\
(30.33)\end{array}$ & $\begin{array}{c}51.33 \\
(45.76)\end{array}$ & $\begin{array}{l}61.66 \\
(51.75)\end{array}$ & $\begin{array}{l}64.66 \\
(53.5)\end{array}$ & $\begin{array}{c}65.00 \\
( \\
53.72)\end{array}$ & $\begin{array}{c}65.00 \\
( \\
53.72)\end{array}$ & $\begin{array}{c}65.00 \\
( \\
53.72)\end{array}$ & 11.24 \\
\hline $\mathrm{T}_{6}$ & $\begin{array}{c}5.63 \\
(13.55)\end{array}$ & $\begin{array}{c}27.66 \\
(31.64)\end{array}$ & $\begin{array}{c}57.66 \\
(49.41)\end{array}$ & $\begin{array}{l}64.00 \\
(51.13)\end{array}$ & $\begin{array}{c}83.33 \\
(68.91)\end{array}$ & $\begin{array}{l}84.00 \\
(69.35)\end{array}$ & $\begin{array}{c}84.00 \\
(69.35)\end{array}$ & $\begin{array}{l}84.00 \\
(69.35)\end{array}$ & 11.03 \\
\hline $\mathrm{T}_{7}$ & $\begin{array}{c}2.63 \\
(10.75)\end{array}$ & $\begin{array}{c}24.53 \\
(33.21)\end{array}$ & $\begin{array}{l}39.85 \\
(39.44)\end{array}$ & $\begin{array}{c}42.25 \\
(41.44)\end{array}$ & $\begin{array}{l}44.25 \\
(42.44)\end{array}$ & $\begin{array}{c}44.25 \\
(42.44)\end{array}$ & $\begin{array}{c}45.49 \\
(43.21)\end{array}$ & $\begin{array}{c}45.49 \\
(43.21)\end{array}$ & 9.24 \\
\hline $\mathrm{T}_{8}$ & $\begin{array}{c}2.33 \\
(6.75)\end{array}$ & $\begin{array}{c}23.53 \\
(30.21)\end{array}$ & $\begin{array}{c}39.25 \\
(38.44)\end{array}$ & $\begin{array}{c}40.25 \\
(39.44)\end{array}$ & $\begin{array}{c}41.25 \\
(40.44)\end{array}$ & $\begin{array}{c}41.25 \\
(40.44)\end{array}$ & $\begin{array}{c}41.25 \\
(40.44)\end{array}$ & $\begin{array}{c}41.25 \\
(42.44)\end{array}$ & 9.01 \\
\hline $\mathrm{T}_{9}$ & $\begin{array}{c}2.23 \\
(5.75)\end{array}$ & $\begin{array}{c}22.53 \\
(28.21)\end{array}$ & $\begin{array}{l}37.25 \\
(37.44)\end{array}$ & $\begin{array}{l}37.25 \\
(37.44)\end{array}$ & $\begin{array}{c}37.25 \\
(37.44)\end{array}$ & $\begin{array}{c}37.25 \\
(37.44)\end{array}$ & $\begin{array}{c}37.25 \\
(37.44)\end{array}$ & $\begin{array}{c}37.25 \\
(37.44)\end{array}$ & 8.25 \\
\hline $\mathrm{T}_{10}$ & $\begin{array}{c}2.53 \\
(8.75)\end{array}$ & $\begin{array}{c}20.53 \\
(23.21)\end{array}$ & $\begin{array}{c}33.25 \\
(32.44)\end{array}$ & $\begin{array}{c}43.12 \\
(39.21)\end{array}$ & $\begin{array}{c}48.00 \\
( \\
41.04)\end{array}$ & $\begin{array}{c}49.54 \\
(42.32)\end{array}$ & $\begin{array}{l}50.74() \\
(43.49)\end{array}$ & $\begin{array}{c}50.74 \\
(43.49)\end{array}$ & 15.52 \\
\hline $\mathrm{CD}_{0.5}$ & 2.01 & 3.24 & 3.11 & 2.89 & 2.75 & 3.01 & 3.01 & 3.01 & 2.36 \\
\hline
\end{tabular}

Note: Data in parentheses are average transformed value.
$\mathrm{T}_{1}=(24 \mathrm{hrs}+100 \mathrm{ppmGA} 3)$
$\mathrm{T}_{2}=(24 \mathrm{hrs}+200 \mathrm{ppmGA} 3)$
$\mathrm{T}_{3}=(24 \mathrm{hrs}+300 \mathrm{ppmGA} 3)$
$\mathrm{T}_{4}=(48 \mathrm{hrs}+100 \mathrm{ppmGA} 3)$
$\mathrm{T}_{5}=(48 \mathrm{hrs}+200 \mathrm{ppmGA} 3)$
$\mathrm{T}_{6}=(48 \mathrm{hrs}+300 \mathrm{ppmGA} 3)$
$\mathrm{T}_{7}=(72 \mathrm{hrs}+100 \mathrm{ppmGA} 3)$
$\mathrm{T}_{8}=(72 \mathrm{hrs}+200 \mathrm{ppmGA} 3$
$\mathrm{T}_{9}=(72 \mathrm{hrs}+300 \mathrm{ppmGA} 3)$
$\mathrm{T}_{10}=$ (control) 
Influence of stratification duration and hormone concentration in aonla (Emblica officinalis, GAERTN.)

There was insignificant difference on the rate of aonla seed germination as it was conspicuously influenced by duration of stratification and seed treatment. However, the faster rate of seed germination was recorded in $\mathrm{T}_{9} \quad\left(72 \mathrm{hrs}\right.$ stratification $\left.+300 \mathrm{ppm} \quad \mathrm{GA}_{3}\right)$ i.e.8.25 mean days followed by $\mathrm{T}_{8}$ (72 hrs stratification +2 ppm $\mathrm{GA}_{3}$ ) i.e.,9.01 mean days). The slowest rate of seed germination was recorded $\mathrm{T}_{10}$ (control) i.e., 15.52 mean days). Similar result was also recorded by Bahuguna and Pyarelal (1993) in case of Acacia. There was a noticeable and significant effect of treatments on transplanting success. All those treatments respond poor in seed germination also were poor in transplanting success. Though seeds were sown in polythene bags and gently transplanted into the field.

Table: 2 Influence of stratification duration and hormone concentration on seedling mortality and transplanting success in Aonla (Emhlica officinalis, GAERTN.)

\begin{tabular}{|c|c|c|c|c|c|c|}
\hline \multirow[t]{2}{*}{ Treatments } & \multicolumn{5}{|c|}{ Seedling mortality, (\%) } & \multirow{2}{*}{$\begin{array}{l}\text { Transplanting } \\
\text { success, (\%) }\end{array}$} \\
\hline & $\begin{array}{c}28 \\
\text { DAS }\end{array}$ & $\begin{array}{c}35 \\
\text { DAS }\end{array}$ & $\begin{array}{c}42 \\
\text { DAS }\end{array}$ & $\begin{array}{c}49 \\
\text { DAS }\end{array}$ & $\begin{array}{c}56 \\
\text { DAS }\end{array}$ & \\
\hline $\mathrm{T}_{1}$ & $\begin{array}{l}12.00 \\
(22.30)\end{array}$ & $\begin{array}{c}23.09 \\
(32.04)\end{array}$ & $\begin{array}{c}28.93 \\
(34.91)\end{array}$ & $\begin{array}{c}35.01 \\
(38.03)\end{array}$ & $\begin{array}{c}35.01 \\
(38.03)\end{array}$ & 74.43 \\
\hline $\mathrm{T}_{2}$ & $\begin{array}{l}11.00 \\
(21.30) \\
\end{array}$ & $\begin{array}{c}21.09 \\
(30.04) \\
\end{array}$ & $\begin{array}{c}26.93 \\
(32.91) \\
\end{array}$ & $\begin{array}{c}30.91 \\
(34.03) \\
\end{array}$ & $\begin{array}{c}30.01 \\
(34.03) \\
\end{array}$ & 75.25 \\
\hline $\mathrm{T}_{3}$ & $\begin{array}{l}10.99 \\
(21.10) \\
\end{array}$ & $\begin{array}{c}20.89 \\
(29.94) \\
\end{array}$ & $\begin{array}{c}26.63 \\
(32.81) \\
\end{array}$ & $\begin{array}{c}30.01 \\
(33.93) \\
\end{array}$ & $\begin{array}{c}30.01 \\
(33.93) \\
\end{array}$ & 76.01 \\
\hline $\mathrm{T}_{4}$ & $\begin{array}{c}8.99 \\
(20.10) \\
\end{array}$ & $\begin{array}{l}19.99 \\
(29.64) \\
\end{array}$ & $\begin{array}{c}25.66 \\
(31.41)\end{array}$ & $\begin{array}{l}29.00 \\
(33.13)\end{array}$ & $\begin{array}{c}29.00 \\
(33.13) \\
\end{array}$ & 79.40 \\
\hline $\mathrm{T}_{5}$ & $\begin{array}{c}8.63 \\
(19.55)\end{array}$ & $\begin{array}{l}19.66 \\
(28.64)\end{array}$ & $\begin{array}{c}24.66 \\
(30.41)\end{array}$ & $\begin{array}{l}26.00 \\
(32.13)\end{array}$ & $\begin{array}{c}28.00 \\
(32.13)\end{array}$ & 81.24 \\
\hline $\mathrm{T}_{6}$ & $\begin{array}{c}7.63 \\
(15.55)\end{array}$ & $\begin{array}{l}17.66 \\
(24.64)\end{array}$ & $\begin{array}{c}20.66 \\
(27.41)\end{array}$ & $\begin{array}{l}22.00 \\
(28.13)\end{array}$ & $\begin{array}{c}22.00 \\
(28.13)\end{array}$ & 91.03 \\
\hline $\mathrm{T}_{7}$ & $\begin{array}{c}45.63 \\
(39.75)\end{array}$ & $\begin{array}{c}48.53 \\
(40.21)\end{array}$ & $\begin{array}{c}50.85 \\
(43.44)\end{array}$ & $\begin{array}{c}58.25 \\
(52.44)\end{array}$ & $\begin{array}{c}58.25 \\
(52.44)\end{array}$ & 49.24 \\
\hline $\mathrm{T}_{8}$ & $\begin{array}{c}58.33 \\
(51.75) \\
\end{array}$ & $\begin{array}{c}62.53 \\
(55.21) \\
\end{array}$ & $\begin{array}{r}65.25 \\
(57.44) \\
\end{array}$ & $\begin{array}{c}68.25 \\
(58.44) \\
\end{array}$ & $\begin{array}{c}68.25 \\
(58.44) \\
\end{array}$ & 39.01 \\
\hline $\mathrm{T}_{9}$ & $\begin{array}{c}62.23 \\
(55.75)\end{array}$ & $\begin{array}{c}69.53 \\
(58.21)\end{array}$ & $\begin{array}{c}77.25 \\
(62.44)\end{array}$ & $\begin{array}{c}79.25 \\
(65.44)\end{array}$ & $\begin{array}{c}79.25 \\
(65.44)\end{array}$ & 35.25 \\
\hline $\mathrm{T}_{10}$ & $\begin{array}{c}46.63 \\
(39.95)\end{array}$ & $\begin{array}{c}49.53 \\
(41.21)\end{array}$ & $\begin{array}{c}51.85 \\
(44.44)\end{array}$ & $\begin{array}{c}59.25 \\
(53.44)\end{array}$ & $\begin{array}{c}59.25 \\
(53.44)\end{array}$ & 65.52 \\
\hline C.D. at $5 \%$ & 2.31 & 3.54 & 3.42 & 3.89 & 2.95 & 4.43 \\
\hline
\end{tabular}

(Note: figures in parentheses are average transformed value)

The differences due to various treatments in respect of seedling mortality differed significantly. The mortality of aonla seedling ranges between 22.00 to 79.25 per cent within 56 days of seed sowing. The highest mortality was recorded $(79.25 \%)$ in $\mathrm{T}_{9}$ $\left(72 \mathrm{hrs}\right.$ stratification $\left.+300 \mathrm{ppm} \mathrm{GA}_{3}\right)$ followed by 68.25 per cent in $\mathrm{T}_{8} \quad(72 \mathrm{hrs}$ stratification $+200 \mathrm{ppm} \mathrm{GA}_{3}$ ) and the value was lowest $(22.00 \%)$ in $\mathrm{T}_{6} \quad(48 \mathrm{hrs}$ stratification +300 ppm $\mathrm{GA}_{3)}$ treatment. Similar results were also found by Awang and Hamzah (1986) in Acacia. Aonla seed soaking more than 48 houres was proved detrimental in term of seed germination and mortality.

Therefore, soaking hours should not constitute more than 48 hours to achieve better survival of aonla seedlings. Obviously, more leaching had toxic effect of hormone on tender seedlings and higher osmotic pressure, imbalanced nutrient level lead to mortality of the seedlings The findings are in the conformity of the findings of the Shamet et al., (1994) and Gupta (1989).

Based on the result obtained from investigation it can be concluded that seed 
soaking for 48 hours followed by $300 \mathrm{ppm}$ seed treatment with $\mathrm{GA}_{3}$ resulted best performance with regards to percent seed germination $(84.00 \%)$ and least seedling mortality $(22.00 \%)$.

\section{References}

1. Awang, K. and Hamzah, M.B. (1986). Effect of potting mixtures and fertilizers on the growth of Acacia mangium wild seedlings. Malaysian Application Biology, 15 (1): 31-42.

2. Ayers, R.S. and Wescot, D.W. (2006). Water quality of agriculture; irrigation and drainage. Paper, 29: FAO, Rome, P. 97.

3. Bahuguna, V. K. and Pyarelal, 1993, Standardization of nursery techniques of Acacia auriculiformis A. cunn. Ex benth. Under Dehra Dun climatic conditions. Part-II effect of soil medium, containers and seed sowing. Indian Forestry. 119: 211-215.

4. Bisla, S.S., Singhrot, R.S. and Chauhan, K.S. (1984). Effect of growing media and urea application on seed germination and growth of Ber. Haryana Journal of Horticulture Science, 13(3-4): 118-122.

5. Dewey, D. R. (1960). Salt tolerance of twenty five strains of agropyron. Agronomy Journal, 52: 631-635.

6. Govind, S. and Chandra, R. (1993). Standardization of suitable potting media for raising seedlings of Khasi Mandrin. Indian Journal of Horticulture, 50(3):224-227.

7. Gupta, G.N. (1995). Rainwater management for tree planting in Indian desert. Journal of Arid Environment, 31: 219-235.

8. Paliwal, K.V. and Gandhi, A. P. (1968). Anion effect on germination of some jowar and paddy varieties in saline substrate. Indian Journal of Plant Physiology, 11..62-67

9. Shamet, G.S., Chauhan, P.S. and Sood, R. (1994). Nursery studies on potting mixture, mulching and fertilizare requirement on chilgoza pine (Pinus gerardiana Wall.). Indian Journal of Forestry, 17(3): 225-229.

\begin{tabular}{|ll|}
\hline Received & : July, 2017 \\
Revised & : Oct., 2017 \\
Published & : Dec., 2017 \\
\hline
\end{tabular}

